Article

\title{
Experiments on the Performance of Small Horizontal Axis Wind Turbine with Passive Pitch Control by Disk Pulley
}

\author{
Yu-Jen Chen and Y. C. Shiah * \\ Institute of Aeronautics and Astronautics, National Cheng Kung University, Tainan 70101, Taiwan; \\ p48981201@mail.ncku.edu.tw \\ * Correspondence: ycshiah@mail.ncku.edu.tw; Tel.: +886-6275-7575 (ext. 63623) \\ Academic Editor: Frede Blaabjerg \\ Received: 23 March 2016; Accepted: 4 May 2016; Published: 7 May 2016
}

\begin{abstract}
The present work is to design a passive pitch-control mechanism for small horizontal axis wind turbine (HAWT) to generate stable power at high wind speeds. The mechanism uses a disk pulley as an actuator to passively adjust the pitch angle of blades by centrifugal force. For this design, aerodynamic braking is caused by the adjustment of pitch angles at high wind speeds. As a marked advantage, this does not require mechanical brakes that would incur electrical burn-out and structural failure under high speed rotation. This can ensure the survival of blades and generator in sever operation environments. In this paper, the analysis uses blade element momentum theory (BEMT) to develop graphical user interface software to facilitate the performance assessment of the small-scale HAWT using passive pitch control (PPC). For verification, the HAWT system was tested in a full-scale wind tunnel for its aerodynamic performance. At low wind speeds, this system performed the same as usual, yet at high wind speeds, the equipped PPC system can effectively reduce the rotational speed to generate stable power.
\end{abstract}

Keywords: blade element momentum theory; passive pitch control; disk pulley; small horizontal axis wind turbine

\section{Introduction}

Nowadays, it has been widely recognized that wind is one of the promising green energy sources for its non-regional availability. This has driven significant resource efforts devoted to the study of wind energy (e.g., [1-3]). In principal, there are two major classifications for wind turbines, namely vertical axis wind turbine (VAWT) and horizontal axis wind turbine (HAWT). Typically, a VAWT can generate more power at a relatively low wind speed in urban areas compared to the HAWT. Nevertheless, when they both operate at the same wind speed, a HAWT is expected to generate more power since the aerodynamic drag is less and the entire rotation of all blades receives more wind power. Currently, small-scale wind turbines are gaining more attention due to the fact that rising energy costs have driven the consumer to seek alternative solutions of independent power supply. Despite the high efficiency of the HAWT, its main drawback is the potential risk of circuit burnout or structural failure when the wind speed exceeds its critical value. The present research is to provide a solution to this problem of the small-scale HAWT by an innovative passive pitch-control mechanism.

Over the years, there are various solutions proposed, including the short-circuit braking system, Choudhry et al. [4] has outlined the control requirements for dynamic stall [5] for wind turbines, where three passive control methodologies have been investigated. By applying this approach of dynamic stall control, Yen and Ahmed [6] showed synthetic jet actuation to be effective for a vertical axis wind turbine (VAWT). By the stall-controlled approach, the system may sustain its stable operation 
generating power under a critical wind speed. However, when operating beyond the critical wind speed, the system will be at the risk of over-running due to a rapid rise of receiving excessive wind power. Another approach proposed by Xie et al. [7,8] was to design a folding blade of turbine rotor, controlled by a servo motor [7] or a passive mechanism regulation [8]. The main drawback of this design is its difficulty of retaining dynamic equilibrium at variable speeds of rotation. Another innovative design of a flywheel rotor system was proposed by Jauch and Hippel [9] to control the rotation of HAWT via inertia effect. Still, this flywheel mechanism has very limited capability of braking when the HAWT is subjected to very high wind speeds. Having borrowed the design of trailing edge flap of airplanes, Barlas et al. [10] used active flaps on a small-scale HAWT. An alternative design to protect the HAWT system is the so called "yaw control" mechanism, which applies the principal of misalignment to achieve the goal of reducing rotational speeds. Under operations at high wind speeds, the system shall keep yawing to seek a balance with the incoming wind. Among many innovations in this category, Kragh [11] proposed a rotor speed-dependent yaw control of wind turbines. Although applicable, this design has a potential problem of structural fatigue occurring at its support due to the dynamic loads arising from the frequent oscillations of the system. For this, Ekelund [12] presented mathematical models obtained from the equations of motion and proposed to use a yaw servo for attenuation of structural dynamic load oscillations. Additionally, Shariatpanah [13] developed another model for PMSG-based wind turbine with yaw control. Despite its effectiveness, the active control system requires a complicated design of an electro-mechanical sub-system and thus, the system reliability is still an issue when operated under long lasting sever conditions. Some other active pitch-angle control can be found in [14-17]. Still, these systems require relatively delicate electro-mechanical systems for fulfilling the function of active control and its reliability is always an issue of concerns. Moreover, the issue of consuming extra electric power is another drawback of the consumer's concern for using the active control. To the authors' personal opinions, designs of passive pitch-angle control on these aspects are comparably more ideal in consideration of system reliability and saving of electricity. $\mathrm{Li}$ [18] presented a self-powered passive adaptive control to adjust pitch angle of blade. The principal of the self-powered passive adaptive control is to use aerodynamic force acting upon on the blade as the control power under balance with the spring force. Hertel et al. [19] developed a simple strip theory model for the analysis of a conceptual passive pitch control, providing the aerodynamics as a function of the relative wind vector's magnitude and local angle of attack.

Penghu, the largest island of the Pescadores Islands in the Taiwan Strait, has a wind turbine test unit. Having complete facilities, this platform has acquired the international certification of small wind certification council (SWCC). For collecting data from the original HAWT system without pitch-control, we set up HAWT systems of $200 \mathrm{~W}, 400 \mathrm{~W}$, and $1 \mathrm{~kW}$ output at the Penghu test unit (Figure 1). Figure 2a displays all collected data of the $400 \mathrm{~W}$ system, as an example, for the monitoring period of one month (March 2012). From the collected data, it can be clearly seen that the data meets the designed power under normal operation as shown by the green region (before the rated wind speed). However, the blue region, the wind turbine system is operated over the rated wind speeds and should be braked for protection of over power outputs. Under the overloaded operation of up to $600 \mathrm{~W}$, the long-term operation shall lead to generator corruption (Figure 2b). As the objective of this research, this has inspired us to make the best use of the good wind-farm to stably generate electric power even at over rated wind speeds.

The present work aims to design a disk pulley mechanism for a prototype of a small-scale HAWT system to passively control the blade pitch angle such that the system is capable of outputting stable power at over rated wind speeds. SD8000-airfoil [20] is adopted in the blade design for its excellent lift-to-drag ratio at low Reynolds numbers to acquire a higher power coefficient. This article is divided into three parts: (1) BEMT design and software development; (2) design of the disk pulley mechanism; and (3) wind tunnel experiments. Through the complete processes of design, analysis, and experiments, the feasibility of the prototype is verified at the laboratory stage. This has under laid the foundation of possible extended applications to practical small-scale HAWT systems with various power outputs. 


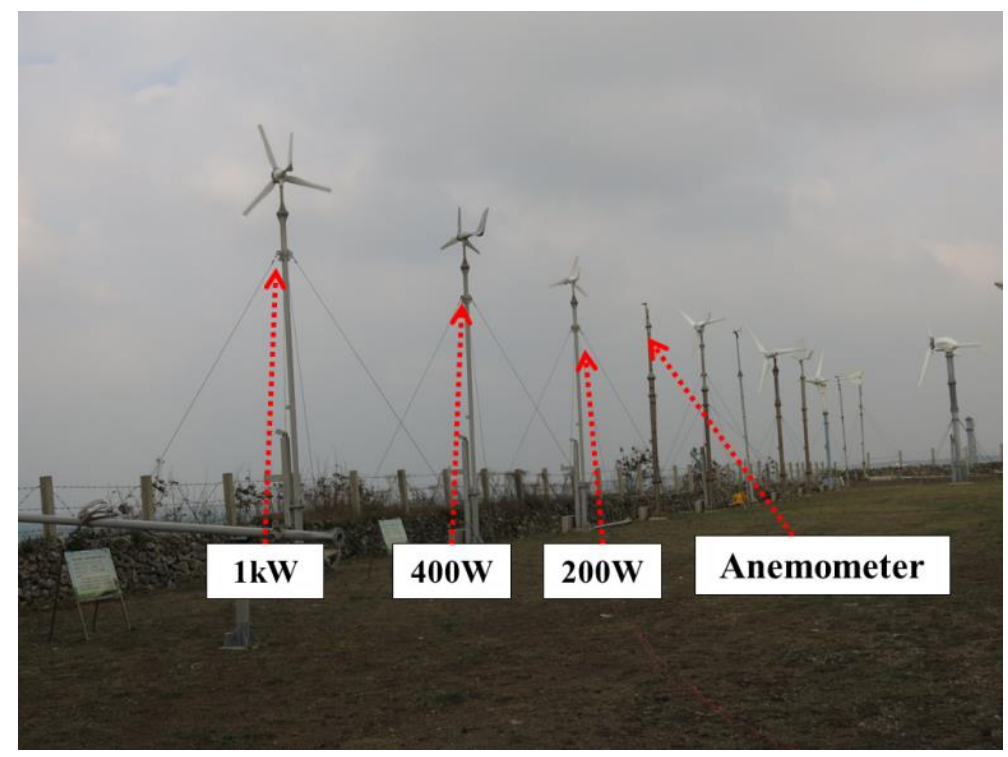

Figure 1. Small-scale HAWT systems tested at Penghu.

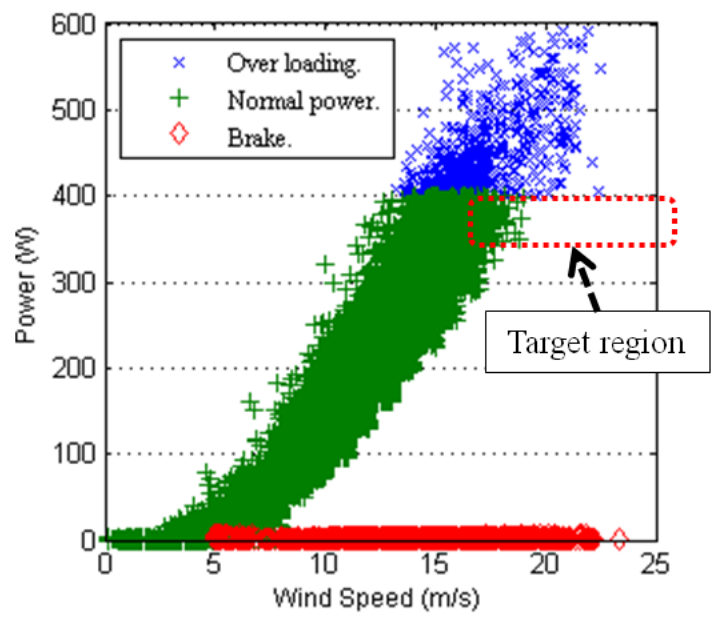

(a)

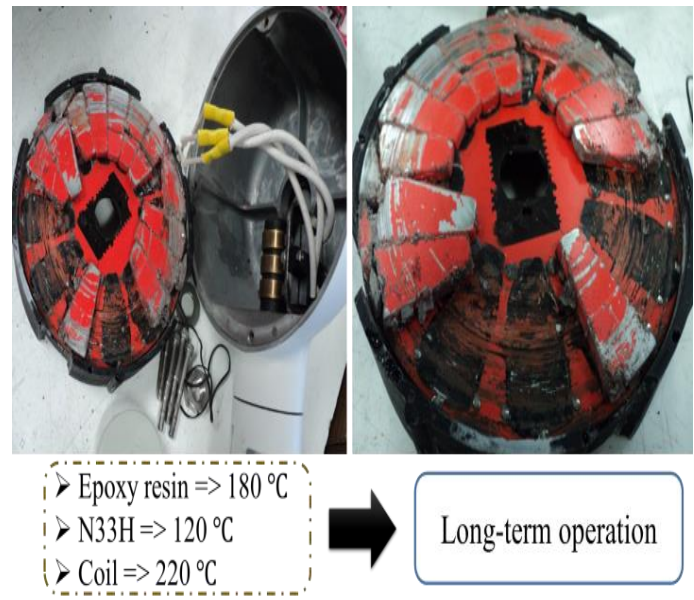

(b)

Figure 2. (a) Monitoring data of the $400 \mathrm{~W}$ HAWT; and (b) damage of the generator of the 400 W HAWT.

\section{Blade Modeling by BEMT and Development of GUI Software}

\subsection{Theoretical Modeling of Blade Design}

Our blade design is based on BEMT, which has been widely applied in industry for the blade design of wind turbines. The flowchart collating the blade design process according to $[21,22]$ is shown in Figure 3a. Table 1 lists all parameters for this blade design. Figure $3 \mathrm{~b}$ shows the prototype and the CAD drawing of the blade design following processes depicted by the flowchart. Table 2 lists all designed values of chord lengths and pitch angles for ten sections of the airfoil SD8000 [20] as shown in Figure 4a. All defined angles are indicated in Figure $4 \mathrm{~b}$, where $a$ is the axial induction factor and $a^{\prime}$ is the angular induction factor. 


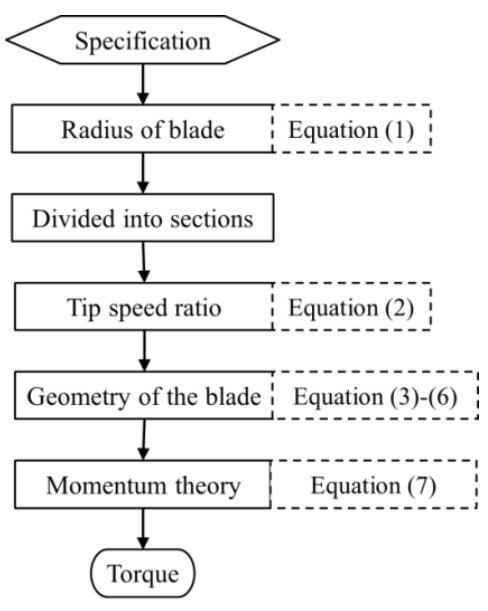

(a)

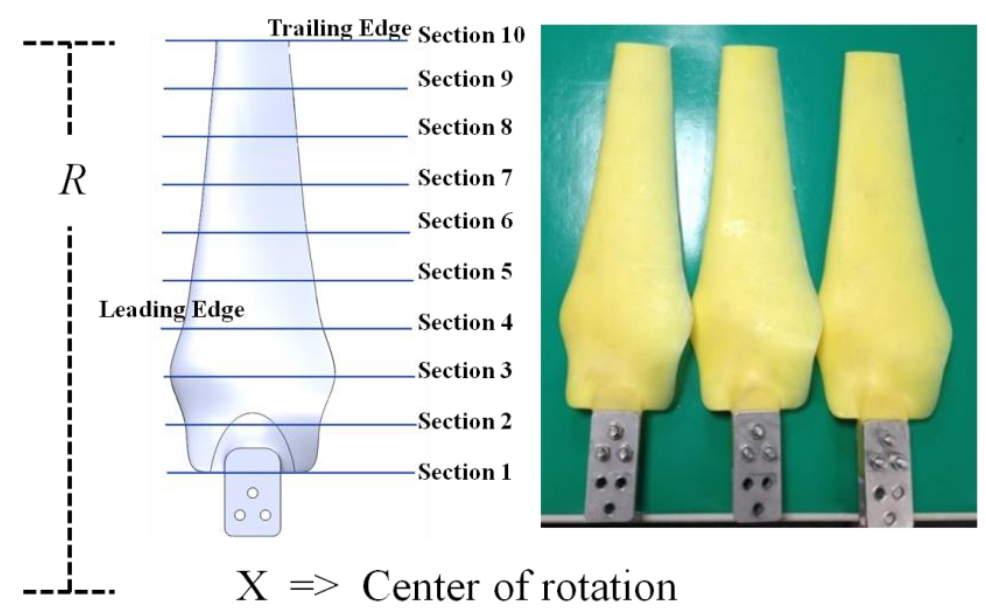

(b)

Figure 3. (a) Flowchart of the blade design from BEMT; and (b) CAD for ten sections of the blade and prototype.

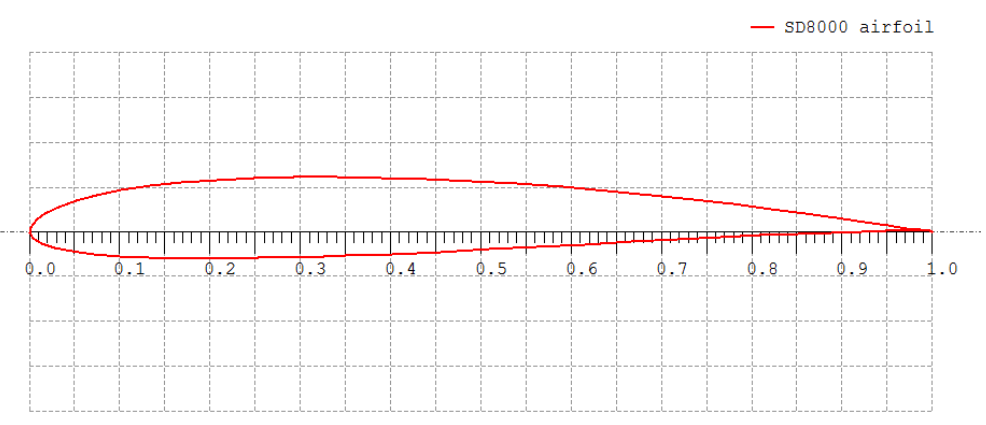

(a)

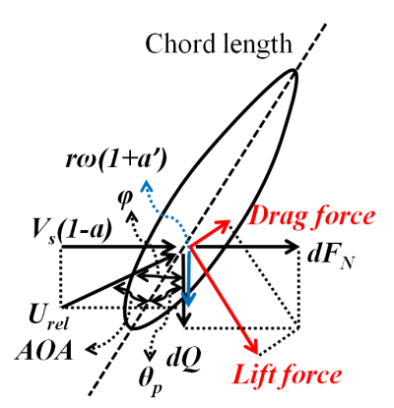

(b)

Figure 4. (a) Geometry of airfoil SD8000; and (b) BEMT angle definition for an airfoil.

Table 1. The parameter of the blade.

\begin{tabular}{cccc}
\hline Item & Parameter & Item & Parameter \\
\hline$\rho$ & $1.225\left(\mathrm{~kg} \cdot \mathrm{m}^{-3}\right)$ & $R$ & $0.41(\mathrm{~m})$ \\
$T S R$ & 3.7 & Root of blade & $0.14(\mathrm{~m})$ \\
$\alpha$ & $5^{\circ}$ & $B$ & 3 \\
$V_{s}$ & $12(\mathrm{~m} / \mathrm{s})$ & Rated Power & $200(\mathrm{~W})$ \\
\hline
\end{tabular}

Table 2. The Geometry parameter of the blade.

\begin{tabular}{cccc}
\hline Section & $\mathbf{r} / \mathbf{R}$ & Chord Length $(\mathbf{m})$ & Pitch Angle (deg) \\
\hline 1 & 0.341 & 0.06 & 0 \\
2 & 0.415 & 0.093 & 18.48 \\
3 & 0.488 & 0.107 & 15.27 \\
4 & 0.561 & 0.095 & 12.81 \\
5 & 0.634 & 0.085 & 10.86 \\
6 & 0.707 & 0.076 & 9.29 \\
7 & 0.780 & 0.070 & 8.00 \\
8 & 0.854 & 0.064 & 6.92 \\
9 & 0.927 & 0.059 & 6.00 \\
10 & 1.000 & 0.055 & 5.21 \\
\hline
\end{tabular}


Principally, the effective radius of rotation $R$ [21-25] is determined by:

$$
R=\sqrt{0.5 \rho V_{s}^{3} C_{p} \pi P}
$$

The tip speed ratio (TSR) of the system, denoted by $\lambda[21-25]$ here, is defined by:

$$
\lambda=\frac{R \omega}{V_{s}}
$$

which is 3.7 for the present design. The speed ratio for each blade section [21-25] is:

$$
\lambda_{r}=\lambda\left(\frac{r}{R}\right)
$$

where $r$ is the rotational radius of each blade section as defined in Table 2. Then, the tip speed ratio at each section given in Equation (3) can be substituted into the following equation to give the local inflow angle by [21-25]:

$$
\varphi=\tan ^{-1}\left(\frac{2}{3 \lambda_{r}}\right)
$$

For giving more detailed derivations, consider the Figure $4 \mathrm{~b}$. From the figure, one may readily have the following relationships from geometrical considerations [21-25]:

$$
\tan \varphi=\frac{V_{s}(1-a)}{\omega r\left(1+a^{\prime}\right)}=\frac{1-a}{\left(1+a^{\prime}\right) \lambda_{r}}
$$

For the case when $\mathrm{a}^{\prime}=0$ (no wake rotation) and $\mathrm{a}=1 / 3$ (the Betz optimum rotor) [21-25], one obtains:

$$
\tan \varphi=\frac{2}{3 \lambda_{r}}
$$

Thus, the values of $\lambda_{r}$ and $\varphi$ obtained above can be further substituted into the following equation to give the corresponding cord length c [21-25]:

$$
c=\frac{8 \pi r \sin \varphi}{3 B C_{L} \lambda_{r}}
$$

For gaining optimal performance, the angle of attack $\alpha$ should be selected to achieve its maximum lift-to-drag ratio. With reference to Figure $4 b$, the pitch angle of blade [21-25] for each section of the rotor is:

$$
\theta_{p}=\varphi-\alpha
$$

A piece of GUI-software, called HAWT-Blade, was developed in MATLAB, where all pertinent formulas in Equations (1)-(8) are integrated altogether, as shown in Figure 5. As can be observed from this figure, the pitch angles and chord lengths near the root are modified for the prototype design. This is mainly due to the large pitch angles and chord lengths near that area, which would cause manufacturing difficulties and overweight of the blade; however, their contributions to the overall resulting torque are very trivial. 


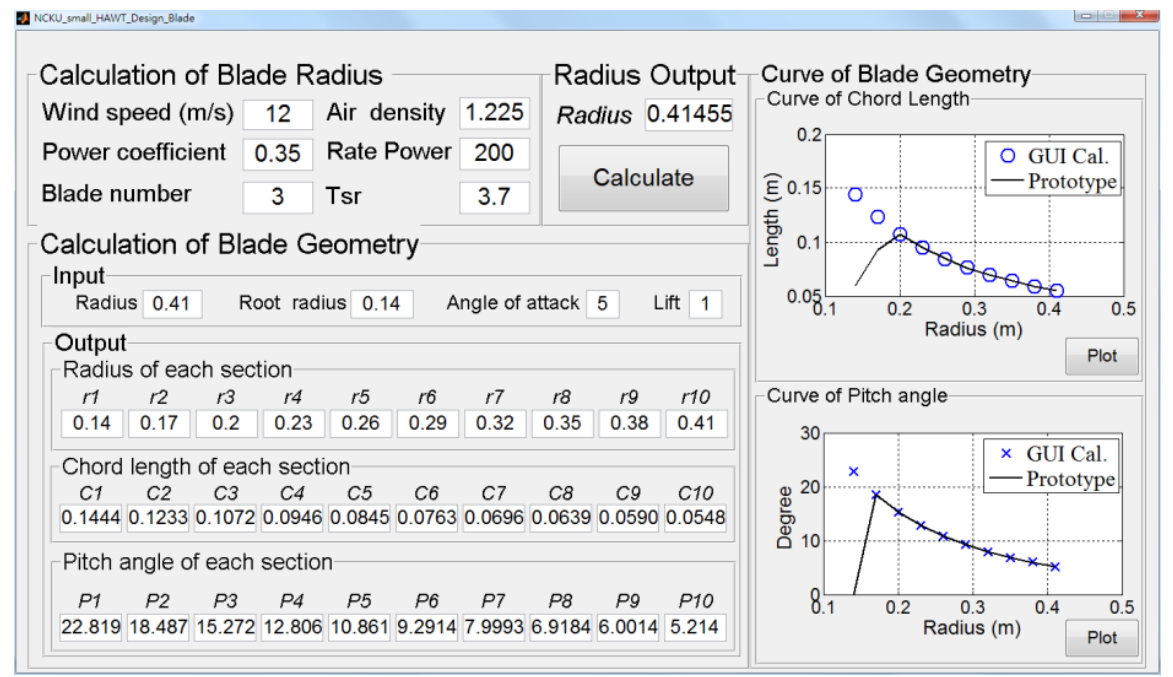

Figure 5. The analysis by HAWT-Blade.

\subsection{GUI of Blade Performance Development}

Having provided all design parameters for the blade design, one may then calculate the resulting torque $Q[21-25]$ by:

$$
Q=\frac{\rho B}{2} U_{r e l}^{2} \Delta \sum_{i=1}^{10}\left(C_{L}^{(i)} \sin \varphi_{i}-C_{D}^{(i)} \cos \varphi_{i}\right) c_{i} r_{i}
$$

where $\Delta$ denotes the constant interval length between two consecutive sections, $C_{L}^{(i)} / C_{D}^{(i)}$ represents the lift/drag coefficient of the $i$-th section, $\phi_{i}$ is the local inflow angle of the $i$-th section, $c_{i}$ and $r_{i}$ stand for the cord length and the rotational radius of the $i$-th section, respectively. Another piece of GUI-software, called HAWT-Perform, was developed using Equation (9) to calculate the output torque. Figure 6 is the GUI of HAWT-Perform, showing such analysis of the resulting torque, output mechanical power, and the corresponding $\mathrm{Cp}$ (power coefficient) of the blade design.

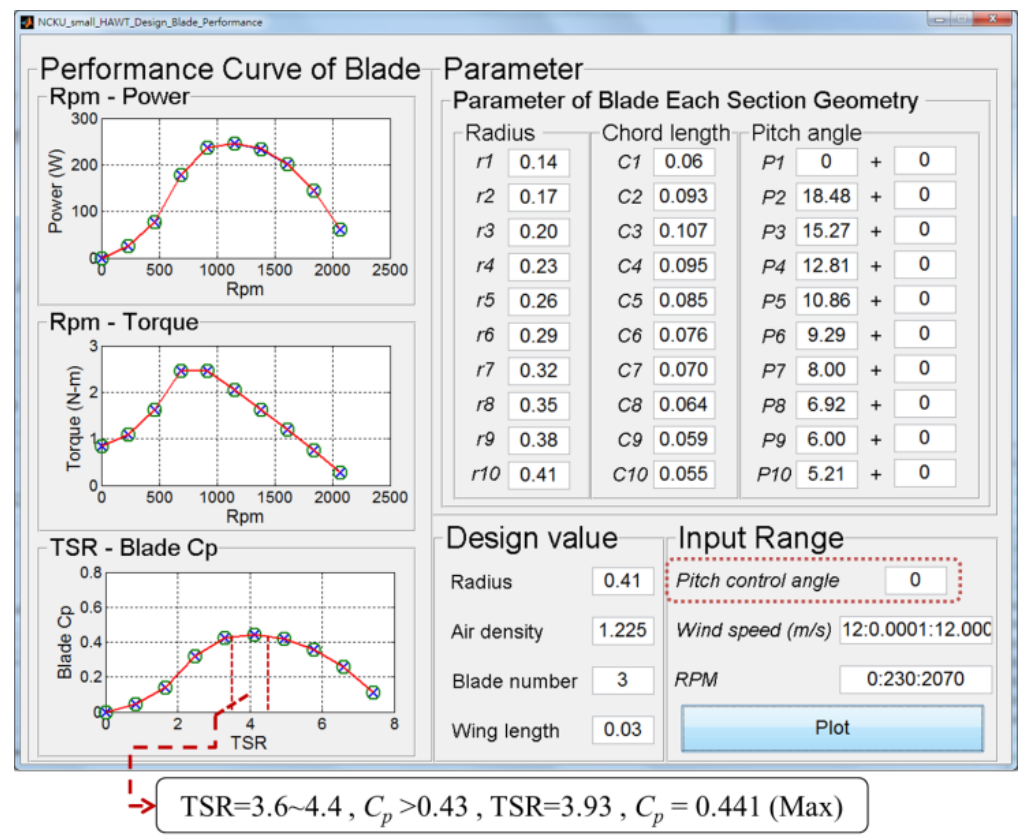

Figure 6. The performance curve of blade predicted by HAWT-Perform. 
As displayed in Figure 6, one may readily obtain corresponding performance curves with an input of pitch control angle for a known geometry of a blade design. From this, it may be easily observed how the variation of pitch control angle affects the resulting performance curves. Just as independent examples for demonstrations, the pitch control angles $0^{\circ} / 10^{\circ} / 20^{\circ} / 30^{\circ}$ were input separately in HAWT-Perform for wind speed equaling $12 \mathrm{~m} / \mathrm{s}$. Each set of outcomes are recorded and plotted in Figure $7 \mathrm{a}, \mathrm{b}$ for the power coefficients and torques, respectively. From these plots, it is evident that the system shall work with reduced torque and power coefficients. The issue remains to design a proper mechanism to adjust pitch control angles at high wind speeds in a passive way. Before elaborating this point of designing the mechanism, it is important to grasp the effect of changing pitch control angle on the resulting flow field around blade, which will be addressed next.

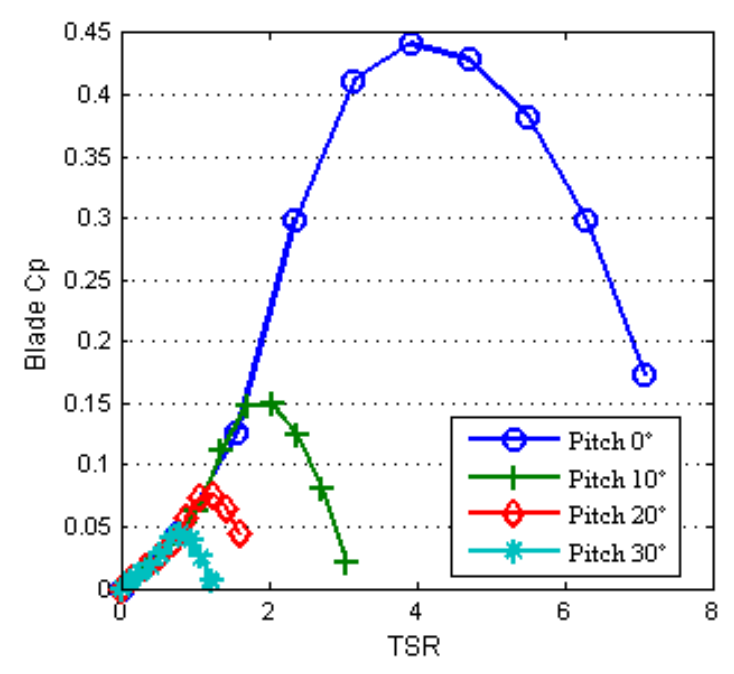

(a)

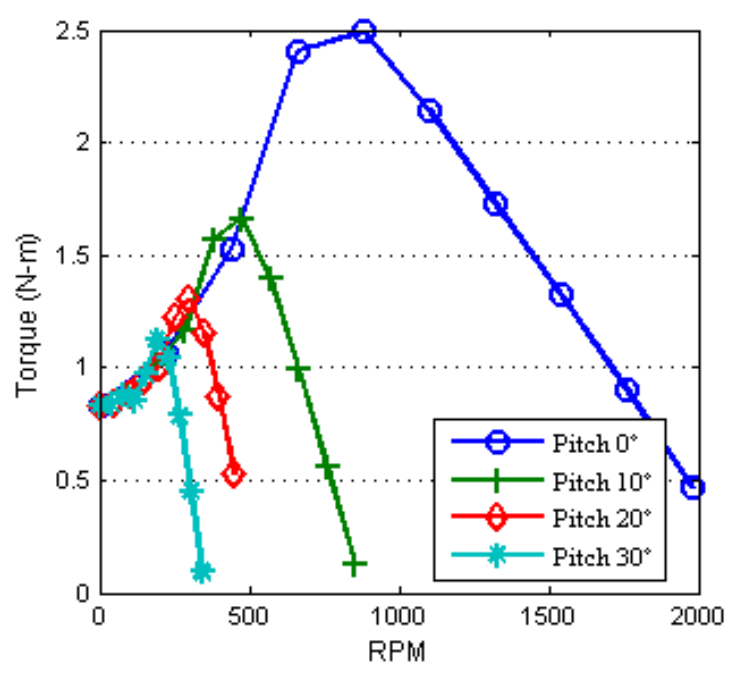

(b)

Figure 7. Performance curves for sample pitch control angles, simulated by HAWT-Perform at $12 \mathrm{~m} / \mathrm{s}$ :

(a) Cp vs. TSR curves; and (b) torque vs. RPM curves.

\section{Mechanical Design of Pitch Control}

In principal, the pitch-control mechanism can be broadly categorized into two kinds: active control and passive control. The former requires delicate electro-mechanical designs with relatively high costs, while the latter is less costly with a simpler design. The most obvious merit of using a passive pitch control is its reliability when operating under sever conditions. There are several designs of passive pitch control proposed over the years. The novel design of this present work employs a disk pulley combined with newly-designed shift-drive components to achieve the goal of passively controlling the pitch angle of turbine blades. In what follows, details of the motion principal along with the functions of each component will be elaborated next.

\subsection{Principle of Motion}

Verifying by the foregoing analyses by theories, one may proceed with the process to design the mechanism of the passive pitch control system. As functioning in motor scooters, the disk pulley can be applied for this purpose. To meet this working requirement, a disk pulley mechanism has been designed as shown in Figure 8a. When in operation, rotors of the blade start to rotate, and as a result, centrifugal forces are produced to drive the disk to move forward (Figure 8a). With the forward moving of the disk pulley at high rotational speeds, pitch angles of the blades are adjusted by an assemblage of bearings. Figure $8 \mathrm{~b}$ shows pictures of the HAWT blades when the disk pulley triggers the rotation at the pitch angle $90^{\circ}$. From Figure $8 \mathrm{~b}$, one may clearly see how the disk pulley functions to control the pitch angle. 


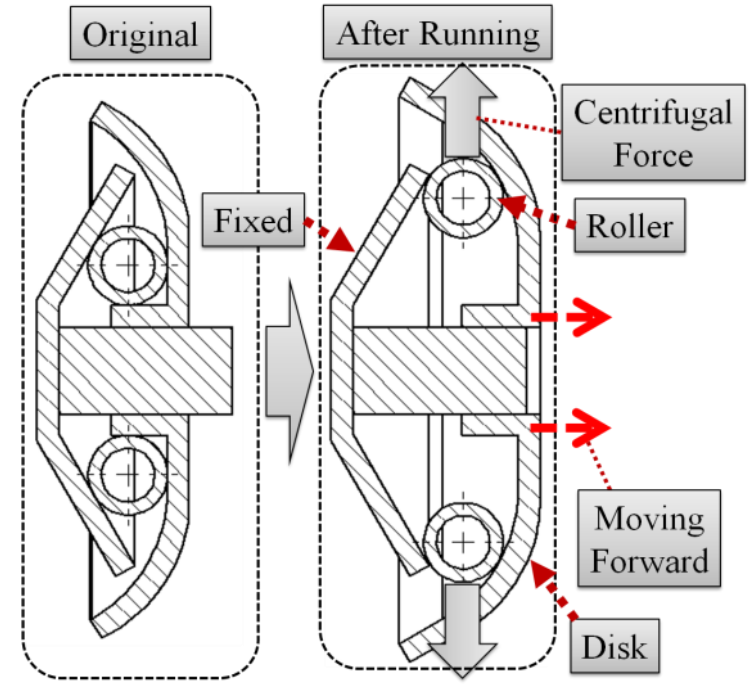

(a)

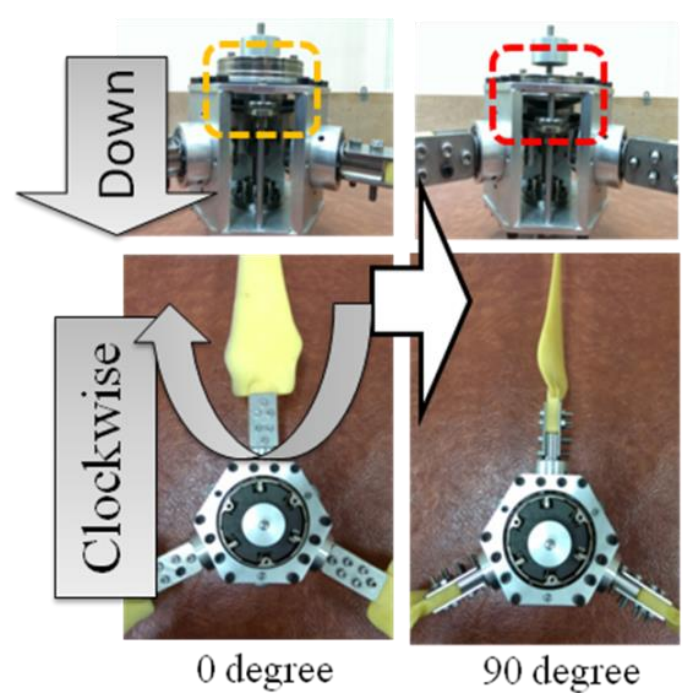

(b)

Figure 8. Mechanism of the passive pitch control: (a) cross-section of the design; and (b) blade motion of the prototype.

\subsection{Components Function}

When the disk pulley operates under high-speed rotation, weight rollers in the disk will be pushed toward the outside wall by the centrifugal force and then drive the blades to rotate by both a cam-bearing and a linear bearing (Figure 9). As displayed in Figure 9, the complete system consists of five main components, namely the disk pulley, bearings, spring, torque sensor, and the blades. In most practice, active pitch control is generally applied with various types of gears powered by servo-motors to change pitch angle (e.g., [7]). This design of power transmission by gears generally faces a problem of assemblage and backlash. To improve the conventional design of gears, this system adopts linear bearings, cam bearings, and ball bearings to alter the direction of movement. The cam bearing is designed to displace along the planned track to drive blades to rotate. Additionally, a spring unit is placed at the center to resist the rigid motion of the disk pulley and to position it back to the original place when the rotation slows down at low rpms.

According to each respective working function, the PPC system mainly consists of four sub-systems, including (1) the pulley system; (2) the forward-moving system; (3) the shift-drive system; and (4) the position-recovering system. For the sub-system (1), the main part is the disk pulley applied in scooters. As shown in Figure 10, there are six weight rollers placed inside the disk. Figure 8a schematically depicts the forward motion of the disk pulley driven by the weight rollers when subjected to high-speed rotation. Being connected to the disk pulley, the linear bearing of the sub-system (2) is, thus, driven to move forward. The sub-system (3) consists of the ball bearing and the cam bearing, functioning to replace the conventional design of a rack and pinion. For this sub-system, the moving force conveyed from (2) is used to turn the rotors and then adjust the pitch angles. By this, the shortcoming of requiring tolerance precision when applying the conventional mechanism of rack and pinion can be overcome. Sub-system (4) includes the components of a spring and an adjustable nut. The spring functions to recover the sub-systems back to their original positions; the nut is used to adjust the resistance force, controlling the triggering speed of rotation for adjusting pitch angle. 


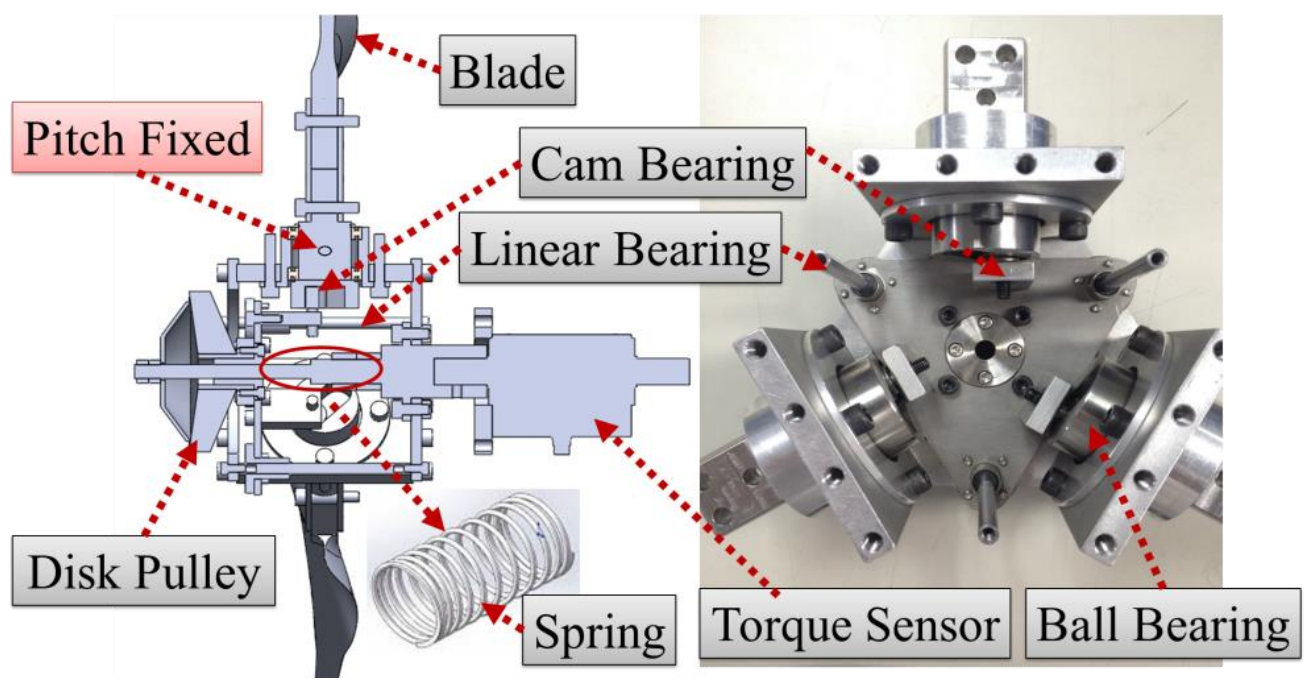

Figure 9. Components of the pitch control mechanism and the prototype.

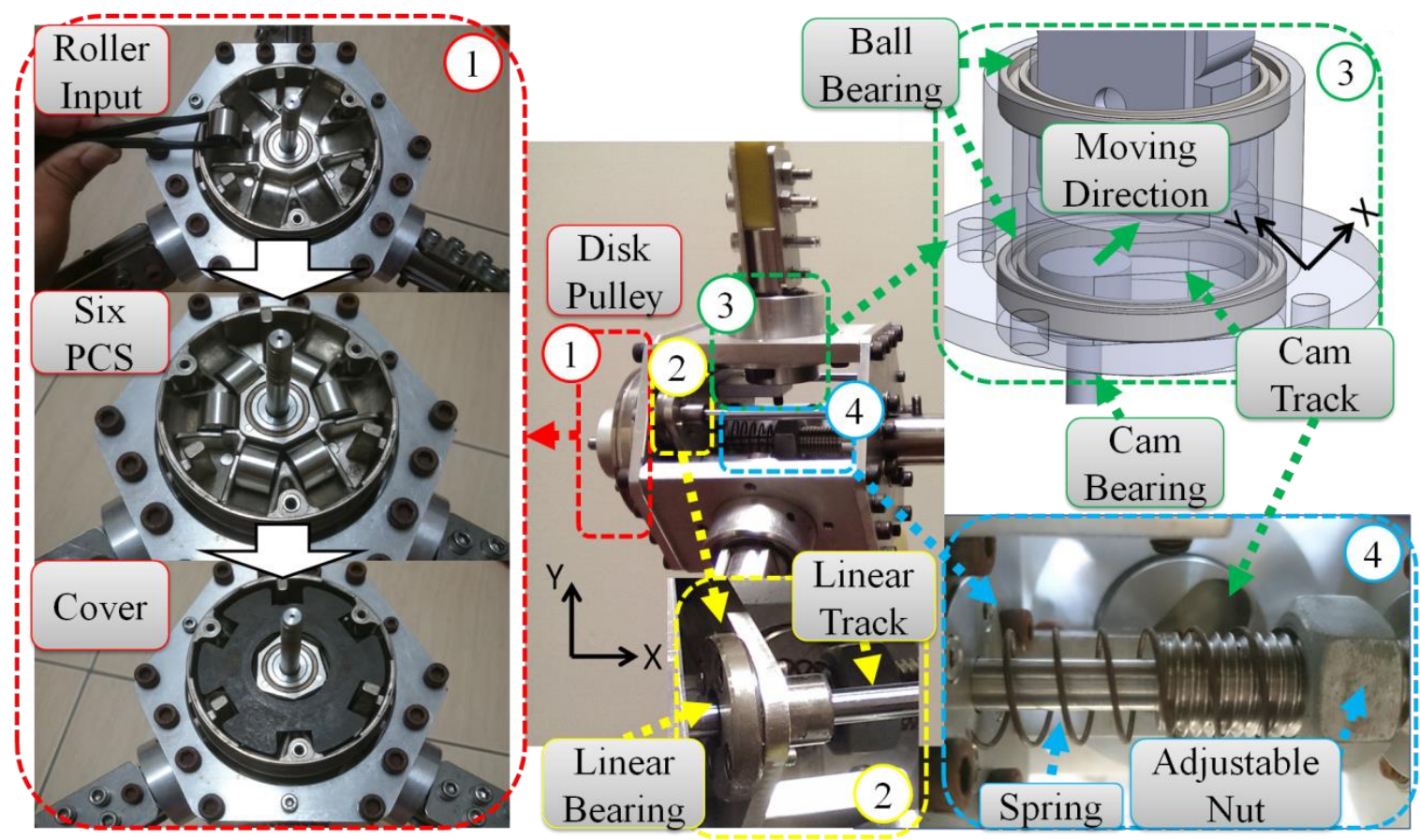

Figure 10. Regional components of the PPC system.

\section{Experiment of ABRI Wind Tunnel}

After confirmation of the performance curves, the wind turbine system was manufactured and experimented in the ABRI (Architecture and Building Research Institute: Tainan, Taiwan) wind tunnel (Figure 11) to measure the generator voltage, current, and torque. The ABRI environment wind tunnel at the National Cheng Kung University (Tainan, Taiwan) was employed to test the aerodynamic performance of the full scale rotor models. This wind tunnel is a closed-circuit type with major components schematically shown in Figure 12. 


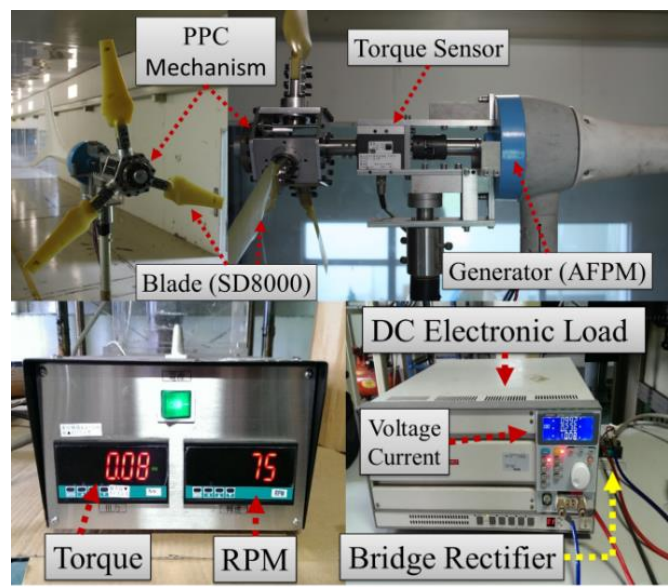

(a)

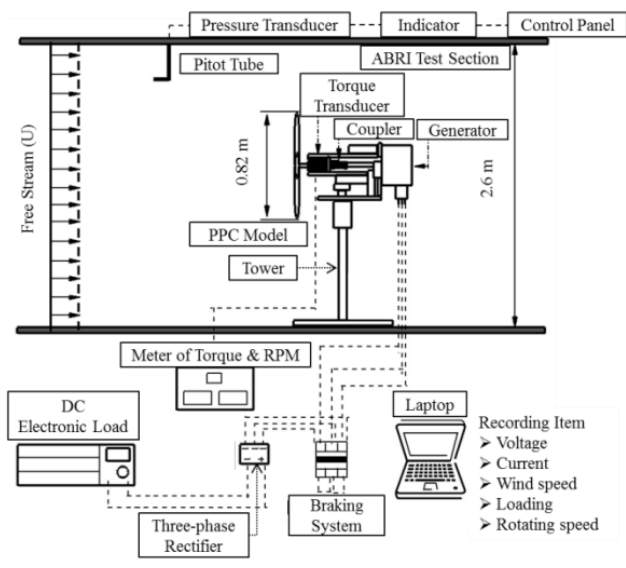

(b)

Figure 11. ABRI wind tunnel tests: (a) the small-scale HAWT prototype and its PPC mechanism; and (b) experimental setups.

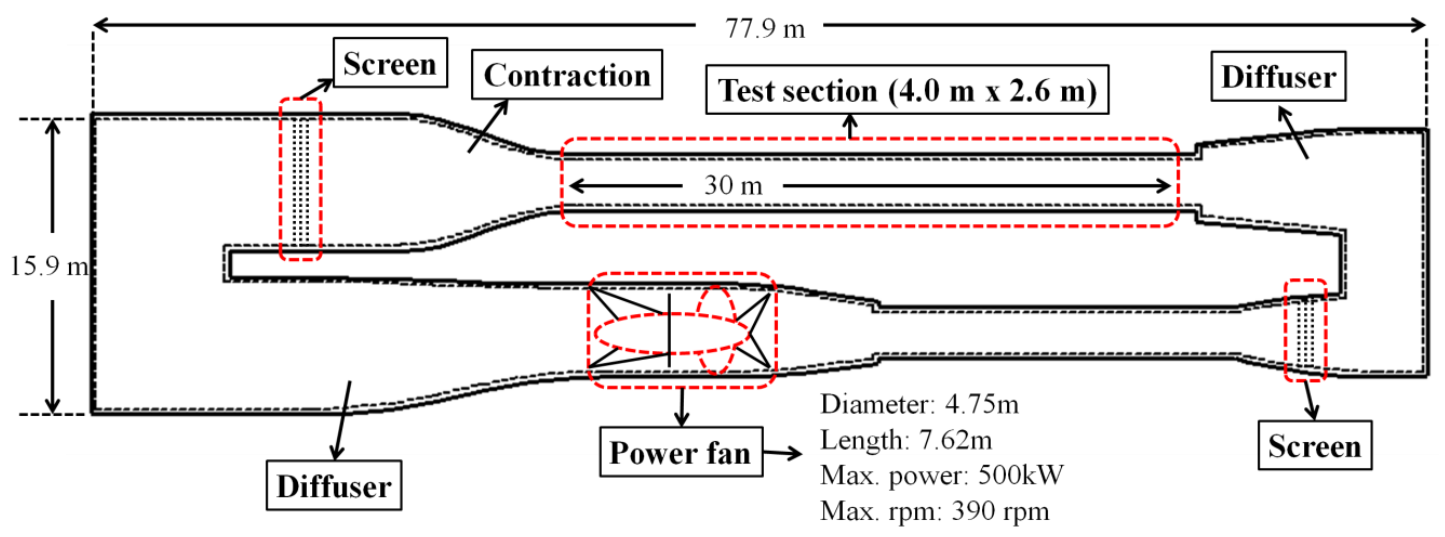

Figure 12. Sketch of the ABRI wind tunnel.

The test section is $36.5 \mathrm{~m}$ long with cross-section dimensions of $4.0 \mathrm{~m} \times 2.6 \mathrm{~m}$. The maximum wind speed in the test section is $36 \mathrm{~m} / \mathrm{s}$. This wind tunnel has been verified for many parameters, including structure vibration, temperature, stability, flow uniformity, turbulence intensity, flow angularity, and boundary layer thickness. The mean flow uniformity is about $0.37 \%$ and the turbulence intensity is less than $0.25 \%$ as well as the boundary layer thickness is about $60 \mathrm{~mm}$ at the inlet cross-section. The average flow angularity of the pitch and yaw angles are, respectively, about $70.415^{\circ}$ and $70.97^{\circ}$ below the wind speed of $20 \mathrm{~m} / \mathrm{s}$. A pitot-static tube was installed to measure the free stream velocity in the test section because it is stable, accurate, and convenient to calibrate. The variable reluctance pressure transducer is connected to the pitot-static tube and converts the pressure difference between the total pressure and the static pressure into electric voltage. The small analog signal was then amplified and converted using an analog/digital converter. The pressure transducer was calibrated by a micro manometer and verified before the experiments. The flow speed in the test section was calculated based on the incompressible steady Bernoulli's equation.

For the prototype to function properly in the ABRI wind tunnel, the choice of $21.5 \mathrm{~g}$ per single weight roller was made and there were six pieces in all. Additionally, the spring coefficient was chosen to be $260(\mathrm{~N} / \mathrm{m})$ as the companioned design parameter. Under these test conditions, the PPC system started to work at the wind speed $8 \mathrm{~m} / \mathrm{s}$ below its rated wind speed $(12 \mathrm{~m} / \mathrm{s})$. For providing comparative data, the same experiment was repeated for the HAWT system, yet with its pitch fixed by a screw (Figure 9), denoted here by PF-system. Since the both experiments of PF/PPC-system were 
conducted for the same prototype, no issue of different hub-weight needs to concern such that the performance difference between the both could be experimentally identified for various wind speeds.

Figure 13 shows the electric output powers and RPM of the both systems at various wind speeds. As can be observed from this figure, both started to generate power at $4 \mathrm{~m} / \mathrm{s}$. Additionally, it is evident to see the difference of the both once the wind speed reached $8 \mathrm{~m} / \mathrm{s}$ when the PPC-system started to work. To our expectation, the PPC-system functioned properly in accordance with the original design whereas, in contrast, the PF-system output power in a manner of cubic growth with the wind speed. At this point now, it is very clear that operation of the small HAWT system is very dangerous, particularly when its electric controller malfunctions; at high wind speeds, the system may either break down with its generator burned out for overloading or fall apart from the great centrifugal force. As can be observed from Figure 13, the PPC-system started functioning to protect the system at wind speed reaching $6 \mathrm{~m} / \mathrm{s}$. Under the no-loading condition, the stable rotation speed of the PPC-system is at $720 \mathrm{rpm}$, when operating with the maximum power, the PPC-system generates stable power at about 500-550 rpm.

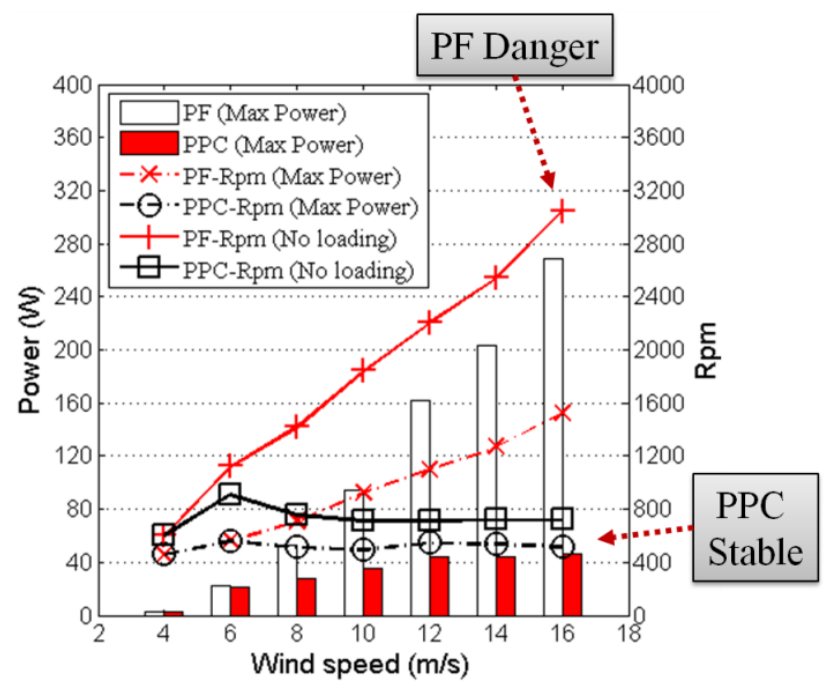

Figure 13. Experiment data of PF and PPC at $4 \mathrm{~m} / \mathrm{s}$ to $16 \mathrm{~m} / \mathrm{s}$.

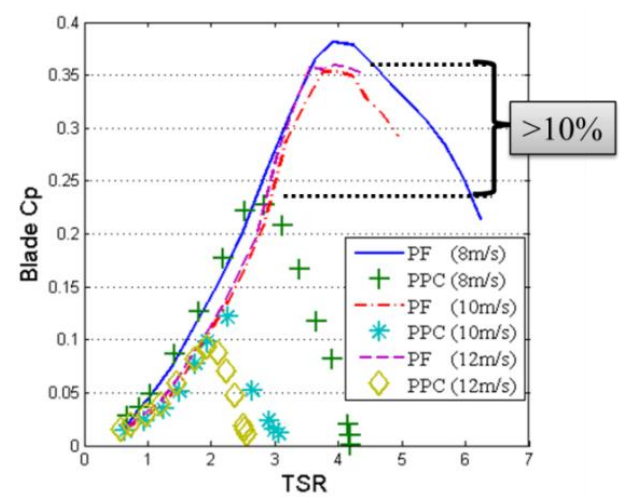

(a)

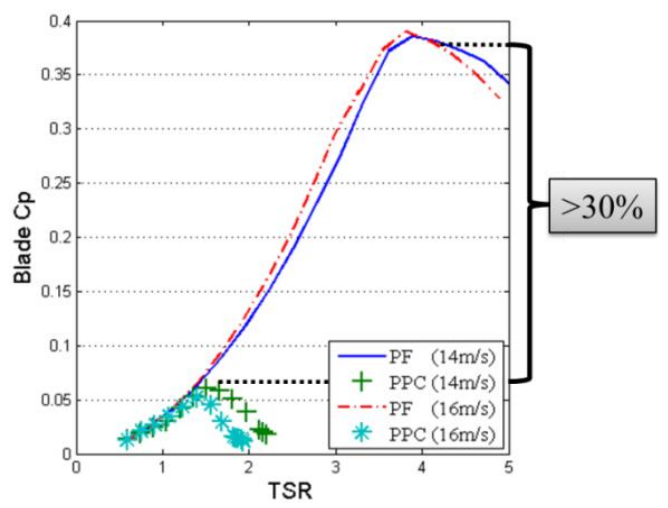

(b)

Figure 14. Experimental results: (a) blade $\mathrm{Cp}-\mathrm{TSR}$ at $8 \mathrm{~m} / \mathrm{s}$ to $12 \mathrm{~m} / \mathrm{s}$; and (b) blade $\mathrm{Cp}-\mathrm{TSR}$ at $14 \mathrm{~m} / \mathrm{s}$ to $16 \mathrm{~m} / \mathrm{s}$.

Figure 14a shows our experimental results for wind speeds at $8 \mathrm{~m} / \mathrm{s}, 10 \mathrm{~m} / \mathrm{s}$, and $12 \mathrm{~m} / \mathrm{s}$; those for wind speeds at $14 \mathrm{~m} / \mathrm{s}$ and $16 \mathrm{~m} / \mathrm{s}$ are in Figure 14b. Obviously, accompanied with the increase of wind speed is the greater percentage of power decrease. At the wind speed $8 \mathrm{~m} / \mathrm{s}$, the maximum 
blade Cp would drop by about $10 \%$ while, at $14 \mathrm{~m} / \mathrm{s}$, the drop would increase up to $30 \%$. From the experiments, the functioning of the PPC system is very clear that it may be applied to protect the system from aerodynamic overloading.

\section{Conclusions}

Under operation at high wind speeds, the small-scale HAWT system will be subjected to aerodynamic overloading that may cause burning out of its generator and/or structural breakdown. There are several techniques to protect the system from the overloading condition. In this article, an innovative disk pulley mechanism is designed to protect the small-scale HAWT system by passive control of the blade pitch angle according to the rotation speed. The theoretical analysis was performed using our self-developed GUI software, called "HAWT-Perform", which can be used to predict the blade performance. Through the theoretical analysis of HAWT-Perform, one may learn the significant performance change when blade pitch angle is adjusted by our disk pulley mechanism. For verification, experiments were carried out for a HAWT prototype with blades (using a SD8000 airfoil) designed by BEMT. From the experiments, one may learn the performance change from the use of our PPC system. This PPC system is featured with the advantages of great reliability, easy maintenance, and low cost. The present work simply determines a feasible approach to protect the small-scale HAWT; however, more extensive studies are required regarding the design parameters of the disk pulley mechanism to match different HAWT systems with other output powers.

Supplementary Materials: The following are available online at www.mdpi.com/link, Video S1: Operation of passive pitch control system.

Acknowledgments: The authors gratefully acknowledge the funding support by National Science Council (NSC 102-2221-E-006-290-MY3). This research is also partially supported by Research Center for Energy Technology and Strategy, National Cheng Kung University and Digisine Energytech Co., Ltd.

Author Contributions: The author Y.C. Shiah planned the experiments and organized the research directions; Yu-Jen Chen performed the mechanism design, theoretical analyses.

Conflicts of Interest: The authors declare no conflict of interest.

\section{Abbreviations}

The following abbreviations are used in this manuscript:

$\begin{array}{ll}\text { NCKU } & \text { National Cheng Kung University } \\ \text { PPC } & \text { Passive pitch control } \\ \text { PF } & \text { Pitch fixed }\end{array}$

\section{References}

1. Mc Garrigle, E.V.; Leahy, P.G. Quantifying the value of improved wind energy forecasts in a pool-based electricity market. Renew. Energy 2015, 80, 517-524. [CrossRef]

2. Browne, O.; Poletti, S.; Young, D. How does market power affect the impact of large scale wind investment in "energy only" wholesale electricity markets? Energy Policy 2015, 87, 17-27. [CrossRef]

3. Gillenwater, M.; Lu, X.; Fischlein, M. Additionality of wind energy investments in the U.S. voluntary green power market. Renew. Energy 2014, 63, 452-457. [CrossRef]

4. Choudhry, A.; Arjomandi, M.; Kelso, R. Methods to control dynamic stall for wind turbine applications. Renew. Energy 2016, 86, 26-37. [CrossRef]

5. Choudhry, A.; Leknys, R.; Arjomandi, M.; Kelso, R. An insight into the dynamic stall lift characteristics. Exp. Thermal Fluid Sci. 2014, 58, 188-208. [CrossRef]

6. Yen, J.; Ahmed, N.A. Enhancing vertical axis wind turbine by dynamic stall control using synthetic jets. J. Wind Eng. Ind. Aerodyn. 2013, 114, 12-17. [CrossRef]

7. Xie, W.; Zeng, P.; Lei, L.P. A novel folding blade of wind turbine rotor for effective power control. Energy Convers. Manag. 2015, 101, 52-65. [CrossRef] 
8. Xie, W.; Zeng, P.; Lei, L.P. Wind tunnel experiments for innovative pitch regulated blade of horizontal axis wind turbine. Energy 2015, 91, 1070-1080. [CrossRef]

9. Jauch, C.; Hippel, S. Hydraulic-pneumatic flywheel system in a wind turbine rotor for inertia control. IET Renew. Power Generat. 2016, 10, 33-41. [CrossRef]

10. Barlas, T.K.; van Wingerden, W.; Hulskamp, A.W.; van Kuik, G.A.M.; Bersee, H.E.N. Smart dynamic rotor control using active flaps on a small-scale wind turbine: Aeroelastic modeling and comparison with wind tunnel measurements. Wind Energy 2013, 16, 1287-1301. [CrossRef]

11. Kragh, K.A.; Fleming, P.A.; Scholbrock, A.K. Increased Power Capture by Rotor Speed-Dependent Yaw Control of Wind Turbines. J. Sol. Energy Eng. 2013, 135, 031018. [CrossRef]

12. Ekelund, T. Yaw control for reduction of structural dynamic loads in wind turbines. J. Wind Eng. Ind. Aerodyn. 2000, 85, 241-262. [CrossRef]

13. Shariatpanah, H.; Fadaeinedjad, R.; Rashidinejad, M. A New Model for PMSG-Based Wind Turbine with Yaw Control. IEEE Trans. Energy Convers. 2013, 28, 929-937. [CrossRef]

14. Lesic, V.; Vasak, M.; Peric, N.; Wolbank, T.M.; Joksimovic, G. Fault-tolerant Control of a Wind Turbine with a Squirrel-cage Induction Generator and Rotor Bar Defects. Automatika 2013, 54, 316-328.

15. Lee, J.; Kim, J.; Kim, Y.H.; Chun, Y.H.; Lee, S.H.; Seok, J.K.; Kang, Y.C. Rotor Speed-based Droop of a Wind Generator in a Wind Power Plant for the Virtual Inertial Control. J. Electr. Eng. Technol. 2013, 8, 1021-1028. [CrossRef]

16. Simley, E.; Pao, L.Y.; Frehlich, R.; Jonkman, B.; Kelley, N. Analysis of light detection and ranging wind speed measurements for wind turbine control. Wind Energy 2014, 17, 413-433. [CrossRef]

17. Chiang, M.H.; Wang, C.S.; Chen, C.S. Intelligent Pitch Control for a 2MW Wind Turbine. Int. J. Fuzzy Syst. 2012, 14, 89-96.

18. Li, S.C. Self-powered passive adaptive control of pitch angle and Betz-shaped wind tunnel. In Proceedings of the International Conference on Renewable Energies and Power Quality, Madrid, Spain, 4-6 May 2010.

19. Hertel, J.; Nygaard, T.; Duque, E. Passive pitch control of small horizontal axis wind turbines. In Proceedings of the 42nd AIAA Aerospace Sciences Meeting and Exhibit, Aerospace Sciences Meetings, Reno, NV, USA, 5-8 January 2004; pp. 2004-1369.

20. Barlow, J.B.; Rae, W.H.; Pope, A. Low-Speed Wind Tunnel Testing, 3rd ed.; Wiley-Interscience: New York, NY, USA, 1999.

21. Burton, T.; Jenkins, N.; Sharpe, D.; Bossanyi, E. Wind Energy Handbook; John Wiley \& Sons Ltd.: Hoboken, NJ, USA, 2001.

22. Manwell, J.F.; McGowan, J.G.; Rogers, A.L. Wind Energy Explained-Theory, Design and Application; John Wiley \& Sons Ltd.: Hoboken, NJ, USA, 2002.

23. Lee, M.-H.; Shiah, Y.C.; Bai, C.-J. Experiments and numerical simulations of the rotor-blade performance for a small-scale horizontal axis wind turbine. J. Wind Eng. Ind. Aerodyn. 2016, 149, 17-29. [CrossRef]

24. Hsiao, F.B.; Bai, C.J.; Chong, W.T. The Performance Test of Three Different Horizontal Axis Wind Turbine (HAWT) Blade Shapes Using Experimental and Numerical Methods. Energies 2013, 6, 2784-2803. [CrossRef]

25. Bai, C.J.; Hsiao, F.B.; Li, M.H.; Huang, G.Y.; Chen, Y.J. Design of 10 kW Horizontal-Axis Wind Turbine (HAWT) Blade and Aerodynamic Investigation Using Numerical Simulation. Procedia Eng. 2013, 67, 279-287. [CrossRef]

(C) 2016 by the authors; licensee MDPI, Basel, Switzerland. This article is an open access article distributed under the terms and conditions of the Creative Commons Attribution (CC-BY) license (http://creativecommons.org/licenses/by/4.0/). 